\title{
On the Analytical and Numerical Solutions in the Quantum Magnetoplasmas: The Atangana Conformable Derivative $(1+3)$-ZK Equation with Power-Law Nonlinearity
}

\author{
Mostafa M. A. Khater $\mathbb{D}^{1},{ }^{1,2}$ Yu-Ming Chu $\mathbb{D}^{3,4}$ Raghda A. M. Attia, ${ }^{1,5}$ Mustafa Inc $\mathbb{D}^{\text {, }}$, \\ and Dianchen $\mathrm{Lu}(\mathbb{D})^{1}$ \\ ${ }^{1}$ Department of Mathematics, Faculty of Science, Jiangsu University, 212013 Zhenjiang, China \\ ${ }^{2}$ Department of Mathematics, El Obour Institutes, 11828 Cairo, Egypt \\ ${ }^{3}$ Department of Mathematics, Huzhou University, Huzhou 313000, China \\ ${ }^{4}$ Hunan Provincial Key Laboratory of Mathematical Modeling and Analysis in Engineering, Changsha University of Science \\ and Technology, Changsha 410114, China \\ ${ }^{5}$ Department of Basic Science, Higher Technological Institute 10th of Ramadan City, El Sharqia 44634, Egypt \\ ${ }^{6}$ Department of Mathematics, Science Faculty, Firat University, 23119 Elazig, Turkey \\ ${ }^{7}$ Department of Medical Research, China Medical University Hospital, China Medical University, Taichung, Taiwan
}

Correspondence should be addressed to Yu-Ming Chu; chuyuming@zjhu.edu.cn and Mustafa Inc; minc@firat.edu.tr

Received 10 June 2020; Accepted 31 July 2020; Published 23 September 2020

Guest Editor: Xiao-Ling Gai

Copyright (c) 2020 Mostafa M. A. Khater et al. This is an open access article distributed under the Creative Commons Attribution License, which permits unrestricted use, distribution, and reproduction in any medium, provided the original work is properly cited.

\begin{abstract}
In this research paper, our work is connected with one of the most popular models in quantum magnetoplasma applications. The computational wave and numerical solutions of the Atangana conformable derivative (1+3)-Zakharov-Kuznetsov (ZK) equation with power-law nonlinearity are investigated via the modified Khater method and septic-B-spline scheme. This model is formulated and derived by employing the well-known reductive perturbation method. Applying the modified Khater $(\mathrm{mK})$ method, septic B-spline scheme to the $(1+3)$-ZK equation with power-law nonlinearity after harnessing suitable wave transformation gives plentiful unprecedented ion-solitary wave solutions. Stability property is checked for our results to show their applicability for applying in the model's applications. The result solutions are constructed along with their $2 \mathrm{D}, 3 \mathrm{D}$, and contour graphical configurations for clarity and exactitude.
\end{abstract}

\section{Introduction}

In the existence of a magnetized e-p-i plasma [1], the ZK equation is one of the widely common methods to characterize the ion-acoustic solitary waves. The magnetized loadvarying dusty plasma is the best location to look for alternate placed dust ion acoustic waves of nonthermal electrons with a vortex-like spread of velocity [2]. In a comprehensive computational analysis, the ZK method was used to spread the dust-acoustic waves in a magnetized dusty plasma [3] and to excite the electrostatic ion-acoustic lone wave in two dimensions of negative ion magnetoplasmas of superthermal electrons [4]. This plasma comprises of nonthermal ions and negatively charged mobile dust crystals, and q-distributed temperature electrons of distinct nonextensivity power [5]. The ZK equation's mathematical formula found by the well-known reductive disruption process [6] is given by

$$
\mathscr{D}_{t}^{\alpha} \mathfrak{B}+\mathfrak{Q} \mathfrak{B} \mathfrak{B}_{z}+\mathfrak{B}_{z z z}+\mathfrak{Q}\left(\mathfrak{B}_{x x z}+\mathfrak{B}_{y y z}\right)=0,
$$

where $\mathscr{D}_{t}^{\alpha}=d^{\alpha} / d t^{\alpha}, 0<\alpha<0, \mathfrak{B}=\mathfrak{B}(x, y, z, t), \quad \mathbb{Q}=2, \quad \mathbb{Q}=$ $1-\left(\mathfrak{H}_{\mathfrak{e}}^{2} / 8\right), \mathfrak{H}_{\mathrm{e}}=\left(\mathscr{H} \sqrt{\omega_{c i} \omega_{c e}}\right) / 2 \mathscr{K}_{B} \mathscr{T}_{F e}, \mathscr{H}=2 \pi, \omega_{c i}=$ $e \mathscr{B}_{0} / 2 \mathscr{K}_{B} \mathscr{T}_{F e}$, and $\omega_{c e}=e \mathscr{B}_{0} / m_{e}, c$. Additionally, $\mathscr{H}$ is Planck's constant; $\sqrt{\omega_{c i} \omega_{c e}}$ is the lower-hybrid resonance frequency; $\omega_{c i}=e \mathscr{B}_{0} / \mathscr{M}_{i} c, \omega_{c e}=e \mathscr{B}_{0} / \mathscr{M}_{e} c$ are the ion 
(electron) gyrofrequency; $\mathscr{M}_{i}$ is the ion mass; and $c$ is the speed of light in vacuum.

Solving this kind of models has attracted many researchers in various areas, chemical physics [7], geochemistry [8], plasma physics [8], fluid mechanics [9], optical fiber [10], solid-state physics [11], and so on [12-15]. Consequently, constructing the exact solutions of these mathematical models is an indispensable tool for detecting novel properties of them that can be used in their various applications. However, finding the exact solutions of them are not easy to process but is also considered a hard and complex process where there is no unified computational or numerical technique that is able to be applied to all nonlinear evolution (NLE) equations. Almost all computational and numerical techniques depend on an auxiliary equation that is considered a pivot tool in these techniques where all obtained solutions via these schemes are special cases of its general solutions [16-24].

For the fractional models, many analytical and numerical methods with various fractional operators have been derived such as the exponential expansion method, Khater method, Kudryashov method, simplest equation method, $\left(\Psi^{\prime} / \Psi\right)$ -expansion method, Riccati expansion method, first integral method, tanh method, and the functional variable method [25-34].

This paper studies the analytical and numerical solutions of the Atangana conformable derivative $(1+3)$-ZK equation with power-law nonlinearity that is given by [35-38].

$$
\mathscr{D}_{t}^{\alpha} \mathfrak{B}+a \mathfrak{B}^{n} \mathfrak{B}_{x}+b\left(\mathfrak{B}_{x x x}+\mathfrak{B}_{y y x}+\mathfrak{B}_{z z x}\right)=0
$$

where $a, b$, respectively, represent the nonlinearity and dispersion real valued constants. Also, $\mathfrak{B}_{t}$ is the evolution term while $n$ represents the power law nonlinearity parameter. Using the following wave transformation $[39,40][\mathfrak{B}=\mathfrak{B}(x$ $\left., y, z, t)=\mathfrak{P}(\mathfrak{F}), \mathfrak{F}=x+y+z+(\lambda / \alpha)(t+(1 / \Gamma(\alpha)))^{\alpha}\right] \quad$ on Equation (1) where $\lambda$ is an arbitrary constant yields

$$
\lambda \mathfrak{P}^{\prime}+\frac{a}{n+1} \mathfrak{P}^{n} \mathfrak{P}^{\prime}+b\left(3 \mathfrak{P}^{\prime \prime}\right)=0
$$

Integrating Equation (3) once with zero constant of the integration leads to

$$
\lambda \mathfrak{P}+\frac{a}{n+1} \mathfrak{P}^{n+1}+3 b \mathfrak{P}^{\prime \prime}=0 .
$$

Through the balancing principle, the terms $\mathfrak{P}^{n+1}$ and $\mathfrak{P}^{\prime \prime}$ force that $m=2 / n$. Thus, we employ another transformation $\mathfrak{P}=\mathfrak{U}^{2 / n}$ on Equation (1) gives

$$
\lambda \mathfrak{U}^{2}+\frac{a}{n+1} \mathfrak{U}^{4}+\frac{3 b(4-2 n)}{n^{2}} \mathfrak{U}^{\prime 2}+\frac{6 b}{n} \mathfrak{U} \mathfrak{U}^{\prime \prime}=0 .
$$

Balancing between the terms of Equation (5) leads to $m=1$.

The outline of this research paper is given as follows. Section 2 employs the $\mathrm{mK}$ method and septic B-spline scheme to get the abundant explicit wave and numerical solutions of the
Atangana conformable derivative $(1+3)$-ZK equation with power-law nonlinearity. Section 3 investigates the stability of the results solutions. Section 4 shows and discusses the obtained results in our research paper. Section 5 gives the graphical demonstration of some of our solutions. Section 6 explains the conclusion of our study.

\section{Implementation}

In this section, we employ three recent analytical schemes to find the explicit wave solutions of the Atangana conformable derivative $(1+3)$-ZK equation with power-law nonlinearity.

2.1. Ion-Acoustic Solitary Waves Solutions. This section gives a transitory elucidation of the $\mathrm{mK}$ method. We now explore a nontrivial solution for Equation (5) in the form

$$
\begin{aligned}
\mathfrak{U} & =\sum_{i=1}^{m} a_{i} \mathscr{K}^{i \mathscr{F}(\mathfrak{\mho})}+\sum_{i=1}^{m} b_{i} \mathscr{K}^{-i \mathscr{F}(\mathfrak{F})}+a_{0} \\
& =a_{1} \mathscr{K}^{\mathscr{F}(\mathfrak{\mho})}+a_{0}+b_{1} \mathscr{K}^{-\mathscr{F}(\mathfrak{F})},
\end{aligned}
$$

where $a_{0}, a_{1}$, and $b_{1}$ are arbitrary constants while $\mathscr{F}(\mathfrak{F})$ is a function that satisfies the next ODE

$$
\mathscr{F}^{\prime}(\mathfrak{F})=\frac{\mathrm{u} \mathscr{K}^{-\mathscr{F}(\mathfrak{F})}+\rho \mathscr{K}^{\mathscr{F}(\mathfrak{F})}+\delta}{\ln (\mathscr{K})}
$$

where $\mathrm{u}, \rho$, and $\delta$ are arbitrary constants. Exchanging the values of $\mathfrak{U}, \mathfrak{U}^{\prime \prime}$ with Equation (6) along (7) and aggregation of all terms with the same power of $\mathscr{K}^{j \mathscr{F}(\mathfrak{F})},(j=-4,-3, \cdots, 3$ , 4) then equating the gathering terms with zero lead to a system of equations. Solving this system yields

Family I

$$
\begin{aligned}
& a_{0} \longrightarrow \frac{a_{1} \sqrt{\delta^{2}-4 \rho \mathrm{u}}+a_{1} \delta}{2 \rho}, \\
& b_{1} \longrightarrow 0, \\
& \lambda \longrightarrow \frac{1}{4}(-3)\left(b \delta^{2}-4 b \rho \mathrm{u}\right), \\
& a \longrightarrow-\frac{9 b \rho^{2}}{4 a_{1}^{2}} \\
& n \longrightarrow-4 .
\end{aligned}
$$

Family II

$$
\begin{aligned}
& a_{0} \longrightarrow \frac{b_{1} \sqrt{\delta^{2}-4 \rho \mathrm{u}}+b_{1} \delta}{2 \mathrm{u}}, \\
& a_{1} \longrightarrow 0, \\
& \lambda \longrightarrow \frac{1}{4}(-3)\left(b \delta^{2}-4 b \rho \mathrm{u}\right), \\
& a \longrightarrow-\frac{9 b \mathrm{u}^{2}}{4 b_{1}^{2}}, \\
& n \longrightarrow-4 .
\end{aligned}
$$


Family III

$$
\begin{aligned}
& a_{1} \longrightarrow \frac{a_{0} \rho}{\delta}, \\
& b_{1} \longrightarrow \frac{a_{0} \mathrm{u}}{\delta}, \\
& \lambda \longrightarrow \frac{1}{4}(-3)\left(b \delta^{2}-4 b \rho \mathrm{u}\right), \\
& a \longrightarrow-\frac{9 b \delta^{2}}{4 a_{0}^{2}}, \\
& n \longrightarrow-4 .
\end{aligned}
$$

Family IV

$$
\begin{aligned}
& a_{0} \longrightarrow \frac{a_{1} \delta}{2 \rho}, \\
& b_{1} \longrightarrow 0, \\
& \lambda \longrightarrow \frac{3}{2}\left(b \delta^{2}-4 b \rho \mathrm{u}\right), \\
& a \longrightarrow-\frac{18 b \rho^{2}}{a_{1}^{2}}, \\
& n \longrightarrow 2 .
\end{aligned}
$$

Family VI

$$
\begin{aligned}
& a_{0} \longrightarrow \frac{b_{1} \delta}{2 \mathrm{u}}, \\
& a_{1} \longrightarrow 0, \\
& \lambda \longrightarrow \frac{3}{2}\left(b \delta^{2}-4 b \rho \mathrm{u}\right), \\
& a \longrightarrow-\frac{18 b \mathrm{u}^{2}}{b_{1}^{2}}, \\
& n \longrightarrow 2 .
\end{aligned}
$$

Thus, using the above families leads to the new exact solitary wave solutions to the Atangana conformable derivative $(1+3)$-ZK equation with power-law nonlinearity in the next formulas.

For $\delta^{2}-4 \rho \mathrm{u}<0, \rho \neq 0$, we get

$$
\begin{aligned}
& \mathfrak{B}_{\mathrm{I}, 1}(x, t)=\frac{\sqrt{2}}{\sqrt{\left(a_{1}\left(\sqrt{4 \rho \mathrm{u}-\delta^{2}} \tan \left(\left(\sqrt{4 \rho \mathrm{u}-\delta^{2}}\left(4 \alpha \mathscr{H}-3 b \phi\left(\delta^{2}-4 \rho \mathrm{u}\right)\right)\right) / 8 \alpha\right)+\sqrt{\delta^{2}-4 \rho \mathrm{u}}\right)\right) / \rho}}, \\
& \mathfrak{B}_{\mathrm{I}, 2}(x, t)=\frac{\sqrt{2}}{\sqrt{\left(a_{1}\left(\sqrt{4 \rho \mathrm{u}-\delta^{2}} \cot \left(\left(\sqrt{4 \rho \mathrm{u}-\delta^{2}}\left(4 \alpha \mathscr{H}-3 b \phi\left(\delta^{2}-4 \rho \mathrm{u}\right)\right)\right) / 8 \alpha\right)+\sqrt{\delta^{2}-4 \rho \mathrm{u}}\right)\right) / \rho}}, \\
& \mathfrak{B}_{\mathrm{II}, 1}(x, t)=\frac{\sqrt{2}}{\sqrt{b_{1}\left(\left(\left(\sqrt{\delta^{2}-4 \rho \mathrm{u}}+\delta\right) / \mathrm{u}\right)-\left(4 \rho /\left(\delta-\sqrt{4 \rho \mathrm{u}-\delta^{2}} \tan \left(\left(\sqrt{4 \rho \mathrm{u}-\delta^{2}}\left(4 \alpha \mathscr{H}-3 b \phi\left(\delta^{2}-4 \rho \mathrm{u}\right)\right)\right) / 8 \alpha\right)\right)\right)\right.}}, \\
& \mathfrak{B}_{\mathrm{II}, 2}(x, t)=\frac{\sqrt{2}}{\sqrt{b_{1}\left(\left(\left(\sqrt{\delta^{2}-4 \rho \mathrm{u}}+\delta\right) / \mathrm{u}\right)-\left(4 \rho /\left(\delta-\sqrt{4 \rho \mathrm{u}-\delta^{2}} \cot \left(\sqrt{4 \rho \mathrm{u}-\delta^{2}}\left(4 \alpha \mathscr{H}-3 b \phi\left(\delta^{2}-4 \rho \mathrm{u}\right)\right) / 8 \alpha\right)\right)\right)\right)}}, \\
& \mathfrak{B}_{\mathrm{III}, 1}(x, t)=\frac{1}{\sqrt{\left(a_{0}\left(\delta^{2}-4 \rho \mathrm{u}\right)\right) /\left(\delta\left(-\sqrt{4 \rho \mathrm{u}-\delta^{2}} \sin \left(\sqrt{4 \rho \mathrm{u}-\delta^{2}}\left(\mathscr{H}-\left(3 b \phi\left(\delta^{2}-4 \rho \mathrm{u}\right) / 4 \alpha\right)\right)\right)+\delta \cos \left(\sqrt{4 \rho \mathrm{u}-\delta^{2}}\left(\mathscr{H}-\left(3 b \phi\left(\delta^{2}-4 \rho \mathrm{u}\right) / 4 \alpha\right)\right)\right)+\delta\right)\right)}}, \\
& \mathfrak{B}_{\mathrm{III}, 2}(x, t)=\frac{1}{\sqrt{-\left(a_{0}\left(\delta^{2}-4 \rho \mathrm{u}\right)\right) /\left(\delta\left(\sqrt{4 \rho \mathrm{u}-\delta^{2}} \sin \left(\sqrt{4 \rho \mathrm{u}-\delta^{2}}\left(\mathscr{H}-\left(3 b \phi\left(\delta^{2}-4 \rho \mathrm{u}\right) / 4 \alpha\right)\right)\right)+\delta \cos \left(\sqrt{4 \rho \mathrm{u}-\delta^{2}}\left(\mathscr{H}-\left(3 b \phi\left(\delta^{2}-4 \rho \mathrm{u}\right) / 4 \alpha\right)\right)\right)-\delta\right)\right)}}, \\
& \mathfrak{B}_{\mathrm{IV}, 1}(x, t)=\frac{a_{1} \sqrt{4 \rho \mathrm{u}-\delta^{2}} \tan \left(\left(\sqrt{4 \rho \mathrm{u}-\delta^{2}}\left(3 b \phi\left(\delta^{2}-4 \rho \mathrm{u}\right)+2 \alpha \mathscr{H}\right)\right) / 4 \alpha\right)}{2 \rho}, \\
& \mathfrak{B}_{\mathrm{IV}, 2}(x, t)=\frac{a_{1} \sqrt{4 \rho \mathrm{u}-\delta^{2}} \cot \left(\left(\sqrt{4 \rho \mathrm{u}-\delta^{2}}\left(3 b \phi\left(\delta^{2}-4 \rho \mathrm{u}\right)+2 \alpha \mathscr{H}\right)\right) / 4 \alpha\right)}{2 \rho}, \\
& \mathfrak{B}_{\mathrm{V}, 1}(x, t)=\frac{1}{2} b_{1}\left(\frac{\delta}{\mathrm{u}}-\frac{4 \rho}{\delta-\sqrt{4 \rho \mathrm{u}-\delta^{2}} \tan \left(\left(\sqrt{4 \rho \mathrm{u}-\delta^{2}}\left(3 b \phi\left(\delta^{2}-4 \rho \mathrm{u}\right)+2 \alpha \mathscr{H}\right)\right) / 4 \alpha\right)}\right), \\
& \mathfrak{B}_{\mathrm{V}, 2}(x, t)=\frac{1}{2} b_{1}\left(\frac{\delta}{\mathrm{u}}-\frac{4 \rho}{\delta-\sqrt{4 \rho \mathrm{u}-\delta^{2}} \cot \left(\left(\sqrt{4 \rho \mathrm{u}-\delta^{2}}\left(3 b \phi\left(\delta^{2}-4 \rho \mathrm{u}\right)+2 \alpha \mathscr{H}\right)\right) / 4 \alpha\right)}\right) .
\end{aligned}
$$


For $\rho \delta^{2}-4 \rho \mathrm{u}<0, \neq 0$, we get

$$
\begin{aligned}
& \mathfrak{B}_{\mathrm{I}, 3}(x, t)=\frac{\sqrt{2}}{\sqrt{-\left(a_{1} \sqrt{\delta^{2}-4 \rho \mathrm{u}}\left(\tanh \left(\left(\sqrt{\delta^{2}-4 \rho \mathrm{u}}\left(4 \alpha \mathscr{H}-3 b \phi\left(\delta^{2}-4 \rho \mathrm{u}\right)\right)\right) / 8 \alpha\right)-1\right)\right) / \rho}}, \\
& \mathfrak{B}_{\mathrm{I}, 4}(x, t)=\frac{\sqrt{2}}{\sqrt{-\left(a_{1} \sqrt{\delta^{2}-4 \rho \mathrm{u}}\left(\operatorname{coth}\left(\left(\sqrt{\delta^{2}-4 \rho \mathrm{u}}\left(4 \alpha \mathscr{H}-3 b \phi\left(\delta^{2}-4 \rho \mathrm{u}\right)\right)\right) / 8 \alpha\right)-1\right)\right) / \rho}}, \\
& \mathfrak{B}_{\mathrm{II}, 3}(x, t)=\frac{\sqrt{2}}{\sqrt{b_{1}\left(\left(\left(\sqrt{\delta^{2}-4 \rho \mathrm{u}}+\delta\right) / \mathrm{u}\right)-\left(4 \rho /\left(\sqrt{\delta^{2}-4 \rho \mathrm{u}} \tanh \left(\left(\sqrt{\delta^{2}-4 \rho \mathrm{u}}\left(4 \alpha \mathscr{H}-3 b \phi\left(\delta^{2}-4 \rho \mathrm{u}\right)\right)\right) / 8 \alpha\right)+\delta\right)\right)\right)}}, \\
& \mathfrak{B}_{\mathrm{II}, 4}(x, t)=\frac{\sqrt{2}}{\sqrt{b_{1}\left(\left(\left(\sqrt{\delta^{2}-4 \rho \mathrm{u}}+\delta\right) / \mathrm{u}\right)-\left(4 \rho /\left(\sqrt{\delta^{2}-4 \rho \mathrm{u}} \operatorname{coth}\left(\left(\sqrt{\delta^{2}-4 \rho \mathrm{u}}\left(4 \alpha \mathscr{H}-3 b \phi\left(\delta^{2}-4 \rho \mathrm{u}\right)\right)\right) / 8 \alpha\right)+\delta\right)\right)\right)}}, \\
& \mathfrak{B}_{\mathrm{III}, 3}(x, t)=\frac{1}{\sqrt{\left(a_{0}\left(\delta^{2}-4 \rho \mathrm{u}\right)\right) /\left(\delta\left(\sqrt{\delta^{2}-4 \rho \mathrm{u}} \sinh \left(\sqrt{\delta^{2}-4 \rho \mathrm{u}}\left(\mathscr{H}-\left(3 b \phi\left(\delta^{2}-4 \rho \mathrm{u}\right) / 4 \alpha\right)\right)\right)+\delta \cosh \left(\sqrt{\delta^{2}-4 \rho \mathrm{u}\left(\mathscr{H}-\left(3 b \phi\left(\delta^{2}-4 \rho \mathrm{u}\right) / 4 \alpha\right)\right)}\right)+\delta\right)\right)}}, \\
& \mathfrak{B}_{\mathrm{III}, 4}(x, t)=\frac{1}{\sqrt{-\left(a_{0}\left(\delta^{2}-4 \rho \mathrm{u}\right)\right) /\left(\delta\left(\sqrt{\delta^{2}-4 \rho \mathrm{u}} \sinh \left(\sqrt{\delta^{2}-4 \rho \mathrm{u}}\left(\mathscr{H}-\left(3 b \phi\left(\delta^{2}-4 \rho \mathrm{u}\right) / 4 \alpha\right)\right)\right)+\delta \cosh \left(\sqrt{\delta^{2}-4 \rho \mathrm{u}}\left(\mathscr{H}-\left(3 b \phi\left(\delta^{2}-4 \rho \mathrm{u}\right) / 4 \alpha\right)\right)\right)-\delta\right)\right)}} \\
& \mathfrak{B}_{\mathrm{IV}, 3}(x, t)=-\frac{a_{1} \sqrt{\delta^{2}-4 \rho \mathrm{u}} \tanh \left(\left(\sqrt{\delta^{2}-4 \rho \mathrm{u}}\left(3 b \phi\left(\delta^{2}-4 \rho \mathrm{u}\right)+2 \alpha \mathscr{H}\right)\right) / 4 \alpha\right)}{2 \rho}, \\
& \mathfrak{B}_{\mathrm{IV}, 4}(x, t)=-\frac{a_{1} \sqrt{\delta^{2}-4 \rho \mathrm{u}} \operatorname{coth}\left(\left(\sqrt{\delta^{2}-4 \rho \mathrm{u}}\left(3 b \phi\left(\delta^{2}-4 \rho \mathrm{u}\right)+2 \alpha \mathscr{H}\right)\right) / 4 \alpha\right)}{2 \rho}, \\
& \mathfrak{B}_{\mathrm{V}, 3}(x, t)=\frac{1}{2} b_{1}\left(\frac{\delta}{\mathrm{u}}-\frac{4 \rho}{\sqrt{\delta^{2}-4 \rho \mathrm{u}} \tanh \left(\left(\sqrt{\delta^{2}-4 \rho \mathrm{u}}\left(3 b \phi\left(\delta^{2}-4 \rho \mathrm{u}\right)+2 \alpha \mathscr{H}\right)\right) / 4 \alpha\right)+\delta}\right), \\
& \mathfrak{B}_{\mathrm{V}, 4}(x, t)=\frac{1}{2} b_{1}\left(\frac{\delta}{\mathrm{u}}-\frac{4 \rho}{\sqrt{\delta^{2}-4 \rho \mathrm{u}} \operatorname{coth}\left(\left(\sqrt{\delta^{2}-4 \rho \mathrm{u}}\left(3 b \phi\left(\delta^{2}-4 \rho \mathrm{u}\right)+2 \alpha \mathscr{H}\right)\right) / 4 \alpha\right)+\delta}\right) .
\end{aligned}
$$

For $\rho \mathrm{u}>0, \mathrm{u} \neq 0, \rho \neq 0, \delta=0$, we get

$$
\begin{aligned}
& \mathfrak{B}_{\mathrm{I}, 5}(x, t)=\frac{1}{\sqrt{\left(a_{1}(\sqrt{\rho \mathrm{u}} \tan (\sqrt{\rho \mathrm{u}}((3 b \rho \mathrm{u} \phi) / \alpha+\mathscr{H}))+\sqrt{\rho(-\mathrm{u})}) / \rho\right.}}, \\
& \mathfrak{B}_{\mathrm{I}, 6}(x, t)=\frac{1}{\sqrt{\left(a_{1}(\sqrt{\rho(-\mathrm{u})}-\sqrt{\rho \mathrm{u}} \cot (\sqrt{\rho \mathrm{u}}(((3 b \rho \mathrm{u} \phi) / \alpha)+\mathscr{H})))\right) / \rho}}, \\
& \mathfrak{B}_{\mathrm{II}, 5}(x, t)=\frac{1}{\sqrt{\left(b_{1}(\sqrt{\rho \mathrm{u}} \cot (\sqrt{\rho \mathrm{u}}(((3 b \rho \mathrm{u} \phi) / \alpha)+\mathscr{H}))+\sqrt{\rho(-\mathrm{u})})\right) / \mathrm{u}}}, \\
& \mathfrak{B}_{\mathrm{II}, 6}(x, t)=\frac{1}{\sqrt{\left(b_{1}(\sqrt{\rho(-\mathrm{u})}-\sqrt{\rho \mathrm{u}} \tan (\sqrt{\rho \mathrm{u}}(((3 b \rho \mathrm{u} \phi) / \alpha)+\mathscr{H})))\right) / \mathrm{u}}}, \\
& \mathfrak{B}_{\mathrm{IV}, 5}(x, t)=\frac{a_{1} \mathrm{u} \tan (\sqrt{\rho \mathrm{u}}(\mathscr{H}-((6 b \rho \mathrm{u} \phi) / \alpha)))}{\sqrt{\rho \mathrm{u}}}, \\
& \mathfrak{B}_{\mathrm{IV}, 6}(x, t)=-\frac{a_{1} \mathrm{u} \cot (\sqrt{\rho \mathrm{u}}(\mathscr{H}-((6 b \rho \mathrm{u} \phi) / \alpha)))}{\sqrt{\rho \mathrm{u}}}, \\
& \mathfrak{B}_{\mathrm{V}, 5}(x, t)=\frac{b_{1} \rho \cot (\sqrt{\rho \mathrm{u}}(\mathscr{H}-((6 b \rho \mathrm{u} \phi) / \alpha)))}{\sqrt{\rho \mathrm{u}}}, \\
& \mathfrak{B}_{\mathrm{V}, 6}(x, t)=-\frac{b_{1} \rho \tan (\sqrt{\rho \mathrm{u}}(\mathscr{H}-((6 b \rho \mathrm{u} \phi) / \alpha)))}{\sqrt{\rho \mathrm{u}}} .
\end{aligned}
$$


For $\rho \mathrm{u}<0, \mathrm{u} \neq 0, \rho \neq 0, \delta=0$, we get

$$
\begin{aligned}
& \mathfrak{B}_{\mathrm{I}, 7}(x, t)=\frac{1}{\sqrt{\left(a_{1} \mathrm{u}(\tanh (\sqrt{\rho(-\mathrm{u})}((3 b \rho \mathrm{u} \phi / \alpha)+\mathscr{H}))-1)\right) / \sqrt{\rho(-\mathrm{u})}}}, \\
& \mathfrak{B}_{\mathrm{I}, 8}(x, t)=\frac{1}{\sqrt{\left(a_{1} \mathrm{u}(\operatorname{coth}(\sqrt{\rho(-\mathrm{u})}((3 b \rho \mathrm{u} \phi / \alpha)+\mathscr{H}))-1)\right) / \sqrt{\rho(-\mathrm{u})}}},
\end{aligned}
$$

$$
\mathfrak{B}_{\mathrm{II}, 7}(x, t)=\frac{1}{\sqrt{\left(b_{1} \sqrt{\rho(-\mathrm{u})}(\operatorname{coth}(\sqrt{\rho(-\mathrm{u})}((3 b \rho \mathrm{u} \phi / \alpha)+\mathscr{H}))+1)\right) / \mathrm{u}}},
$$

$$
\mathfrak{B}_{\mathrm{II}, 8}(x, t)=\frac{1}{\sqrt{\left(b_{1} \sqrt{\rho(-\mathrm{u})}(\tanh (\sqrt{\rho(-\mathrm{u})}((3 b \rho \mathrm{u} \phi / \alpha)+\mathscr{H}))+1)\right) / \mathrm{u}}},
$$

$$
\mathfrak{B}_{\mathrm{IV}, 7}(x, t)=\frac{a_{1} \mathrm{u} \tanh (\sqrt{\rho(-\mathrm{u})}(\mathscr{H}-(6 b \rho \mathrm{u} \phi) / \alpha))}{\sqrt{\rho(-\mathrm{u})}},
$$

$$
\mathfrak{B}_{\mathrm{IV}, 8}(x, t)=\frac{a_{1} \mathrm{u} \operatorname{coth}(\sqrt{\rho(-\mathrm{u})}(\mathscr{H}-(6 b \rho \mathrm{u} \phi) / \alpha))}{\sqrt{\rho(-\mathrm{u})}},
$$$$
\mathfrak{B}_{\mathrm{V}, 7}(x, t)=\frac{b_{1} \sqrt{\rho} \cot (\sqrt{\rho} \sqrt{\mathrm{u}}(\mathscr{H}-(6 b \rho \mathrm{u} \phi) / \alpha))}{\sqrt{\mathrm{u}}},
$$

$$
\mathfrak{B}_{\mathrm{V}, 8}(x, t)=\frac{b_{1} \sqrt{\rho(-\mathrm{u})} \tanh (\sqrt{\rho(-\mathrm{u})}(\mathscr{H}-\mathscr{H}-(6 b \rho \mathrm{u} \phi) / \alpha))}{\mathrm{u}} .
$$

For $\delta=\mathrm{u} / 2=\kappa, \rho=0$, we get

$$
\begin{aligned}
& \mathfrak{B}_{\mathrm{II}, 9}(x, t)=\frac{2}{\left.\left.\left.\sqrt{b_{1}\left(\left(4 /\left(e^{\kappa \mathscr{H}}-\left(\left(3 b \kappa^{3} \phi\right) / 4 \alpha\right)\right.\right.\right.}-2\right)\right)+\left(\kappa / \sqrt{\kappa^{2}}\right)+1\right)} \\
& \mathfrak{B}_{\mathrm{III}, 9}(x, t)=\frac{1}{\sqrt{a_{0}\left(2 /\left(e^{\kappa \mathscr{H}-\left(\left(3 b \kappa^{3} \phi\right) / 4 \alpha\right)}-2\right)+1\right)}}, \\
& \mathfrak{B}_{\mathrm{VI}, 9}(x, t)=\frac{1}{4} b_{1}\left(\frac{4}{e^{\left(\left(3 b k^{3} \phi\right) / 2 \alpha\right)+\kappa \mathscr{H}}-2}+1\right) .
\end{aligned}
$$

For $\delta=\rho=\kappa, \mathrm{u}=0$, we get

$$
\begin{aligned}
& \mathfrak{B}_{\mathrm{I}, 10}(x, t)=\frac{\sqrt{2}}{\sqrt{a_{1}\left(\left(\kappa / \sqrt{\kappa^{2}}\right)-\operatorname{coth}\left((\kappa \mathscr{H} / 2)-\left(\left(3 b \kappa^{3} \phi\right) / 8 \alpha\right)\right)\right)}}, \\
& \mathfrak{B}_{\mathrm{III}, 10}(x, t)=\frac{1}{\sqrt{-a_{0} / e^{\kappa \mathscr{H}-\left(\left(3 b \kappa^{3} \phi\right) / 4 \alpha\right)}-1}}, \\
& \mathfrak{B}_{\mathrm{IV}, 10}(x, t)=-\frac{1}{2} a_{1} \operatorname{coth}\left(\frac{3 b \kappa^{3} \phi}{4 \alpha}+\frac{\kappa \mathscr{H}}{2}\right) .
\end{aligned}
$$

For $\mathrm{u}=0, \delta \neq 0, \rho \neq 0$, we get

$$
\begin{aligned}
& \mathfrak{B}_{\mathrm{I}, 11}(x, t)=\frac{\sqrt{2}}{\sqrt{\left(a_{1}\left(\delta\left(-4 /\left(\rho e^{\delta \mathscr{H}-\left(3 b \delta^{3} \phi\right) / 4 \alpha}-2\right)-1\right)+\sqrt{\delta^{2}}\right)\right) / \rho}}, \\
& \mathfrak{B}_{\mathrm{III}, 10}(x, t)=\frac{1}{\sqrt{2} \sqrt{-a_{0} /\left(\rho e^{(\delta \mathscr{C}-((3 b \delta \wedge 33 \phi) / 4 \alpha))}-2\right)}}, \\
& \mathfrak{B}_{\mathrm{IV}, 10}(x, t)=\frac{a_{1} \delta\left(4 /\left(2-\rho e^{((3 b \delta \wedge 3 \phi) / 2 \alpha+\delta \mathscr{H})}\right)-1\right)}{2 \rho} .
\end{aligned}
$$

For $\delta=\rho=0, \mathrm{u} \neq 0$, we get

$$
\begin{aligned}
& \mathfrak{B}_{\mathrm{II}, 10}(x, t)=\frac{1}{\sqrt{b_{1} / \lambda t \mathrm{u}+x \mathrm{u}}}, \\
& \mathfrak{B}_{\mathrm{V}, 10}(x, t)=\frac{b_{1}}{\lambda t \mathrm{u}+x \mathrm{u}} .
\end{aligned}
$$

For $\delta=0, \mathrm{u}=\rho$, we get

$$
\begin{aligned}
& \mathfrak{B}_{\mathrm{I}, 11}(x, t)=\frac{1}{\sqrt{a_{1}\left(\tan \left(\left(3 b \mathrm{u}^{3} \phi / \alpha\right)+C+\mathscr{H} u\right)+(\sqrt{-\mathrm{u}} / \sqrt{\mathrm{u}})\right)}}, \\
& \mathfrak{B}_{\mathrm{II}, 11}(x, t)=\frac{1}{\sqrt{b_{1}\left(\cot \left(\left(3 b \mathrm{u}^{3} \phi / \alpha\right)+C+\mathscr{H} u\right)+(\sqrt{-\mathrm{u}} / \sqrt{\mathrm{u}})\right)}}, \\
& \mathfrak{B}_{\mathrm{IV}, 11}(x, t)=\frac{1}{\sqrt{b_{1}\left(\cot \left(\left(3 b \mathrm{u}^{3} \phi / \alpha\right)+C+\mathscr{H} u\right)+(\sqrt{-\mathrm{u}} / \sqrt{\mathrm{u}})\right)}}, \\
& \mathfrak{B}_{\mathrm{V}, 11}(x, t)=a_{1} \tan \left(-\frac{6 b \mathrm{u}^{3} \phi}{\alpha}+C+\mathscr{H} u\right) \\
& \mathfrak{B}_{\mathrm{V}, 11}(x, t)=b_{1} \cot \left(-\frac{6 b \mathrm{u}^{3} \phi}{\alpha}+C+\mathscr{H} u\right) .
\end{aligned}
$$


For $\rho=0, \delta \neq 0, \mathrm{u} \neq 0$, we get

$$
\begin{gathered}
\mathfrak{B}_{\mathrm{II}, 12}(x, t)=\frac{1}{2} b_{1}\left(\frac{2 \delta}{\delta e^{\delta \mathscr{H}-\left(3 b \delta^{3} \phi / 4 \alpha\right)}-\mathrm{u}}+\frac{\sqrt{\delta^{2}}+\delta}{\mathrm{u}}\right), \\
\mathfrak{B}_{\mathrm{III}, 12}(x, t)=a_{0}\left(\frac{\mathrm{u}}{\delta e^{\delta \mathscr{H}-\left(3 b \delta^{3} \phi / 4 \alpha\right)}-\mathrm{u}}+1\right), \\
\mathfrak{B}_{\mathrm{VI}, 12}(x, t)=\frac{1}{2} b_{1} \delta\left(\frac{2}{\delta e^{\left(3 b \delta^{3} \phi / 2 \alpha\right)+\delta \mathscr{H}}-\mathrm{u}}+\frac{1}{\mathrm{u}}\right) .
\end{gathered}
$$

where $\mathscr{H}=x+y+z, \phi=(t+(1 / \Gamma(\alpha)))^{\alpha}$.

2.2. Numerical Solutions. Here, we use three different analytical solutions Equations (16), (19) and (20) to evaluate the numerical solutions of the Atangana conformable derivative $(1+3)$-ZK equation with power-law nonlinearity. Employing the septic spline technique to Equation (5) with the following conditions $a_{1}=3, a_{0}=-6, a=-5 / 4$, $b=5, b_{1}=0, \delta=0, \lambda=-60, n=-4, \rho=-1, \mathrm{u}=4 \& a_{0}=-25$,

$a_{1}=0, a=-9 / 20, b_{1}=5, b=2, \delta=0, \lambda=-150, n=-4, \rho=25$ , $\mathrm{u}=-1 \& a_{1}=3, a_{0}=0, a=-4, b=2, b_{1}=0, \delta=0, \lambda=108, n$ $=2, \rho=-1$, and $\mathrm{u}=9$ gives its numerical solutions in the next form

$$
\mathfrak{B}(\mathfrak{F})=\sum_{\mathfrak{I}=-1}^{\mathfrak{M}+1} \mathfrak{E}_{\mathfrak{M}} \mathscr{E}_{\mathfrak{M}}
$$

where $c_{\mathfrak{M}}, \mathscr{E}_{\mathfrak{M}}$ follow the next conditions, respectively:

$$
\begin{aligned}
& \mathfrak{L} \mathfrak{B}(\mathfrak{F})=\mathscr{F}\left(\mathfrak{\mho}_{\mathfrak{M}}, \mathfrak{B}\left(\mathfrak{\mho}_{\mathfrak{M}}\right)\right) \text { where }(\mathfrak{M}=0,1, \cdots, n)
\end{aligned}
$$

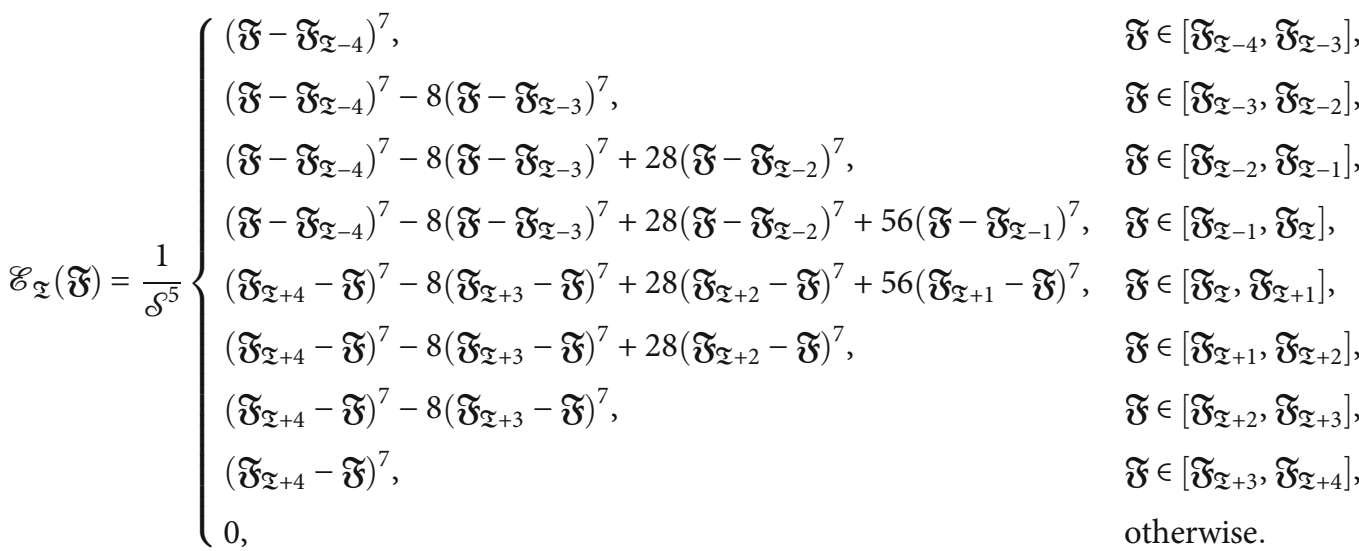

For $\mathfrak{T} \in[-3, \mathfrak{M}+3]$, we get

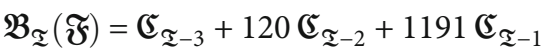

$$
\begin{aligned}
& +2416 \mathfrak{C}_{\mathfrak{I}}+1191 \mathfrak{V}_{\mathfrak{I}+1}+120 \mathfrak{C}_{\mathfrak{I}+2}+\mathfrak{\mathfrak { V }}_{\mathfrak{I}+3} .
\end{aligned}
$$

Substituting Equation (32) into Equation (5) gives $(\mathfrak{M}+7)$ of equations. Resolving this system leads to the following values of exact, numerical, and absolute values or error.

\section{Stability Characteristics}

In this section, the stability property has been tested of the obtained results based on the Hamiltonian system characteristics. This system imposes a single condition to ensure the stability of the solution. This condition is given by

$$
\left.\frac{\partial \mathscr{M}}{\partial \lambda}\right|_{\lambda=\mathscr{G}}>0
$$

where $\mathscr{M}=(1 / 2) \int_{-\mathfrak{E}}^{\mathfrak{E}} \mathfrak{B}^{2} d \mathfrak{F}$ where $\mathfrak{E}$ is an arbitrary constants, $\lambda$ is the frequency, and $\mathscr{G}$ is an arbitrary constant.

Applying the stability check of Equation (20) with the following values of the parameters $a_{1}=3, a_{0}=0, a=-4, b=2$, $b_{1}=0, \delta=0, \lambda=108, n=2, \rho=-1$, and $\mathrm{u}=9$, leads to

$$
\left.\frac{\partial \mathscr{M}}{\partial \lambda}\right|_{\lambda=6}=-2.3447910280083306 \times 10^{-13}<0 .
$$

Consequently, this solution is not stable and applying the 

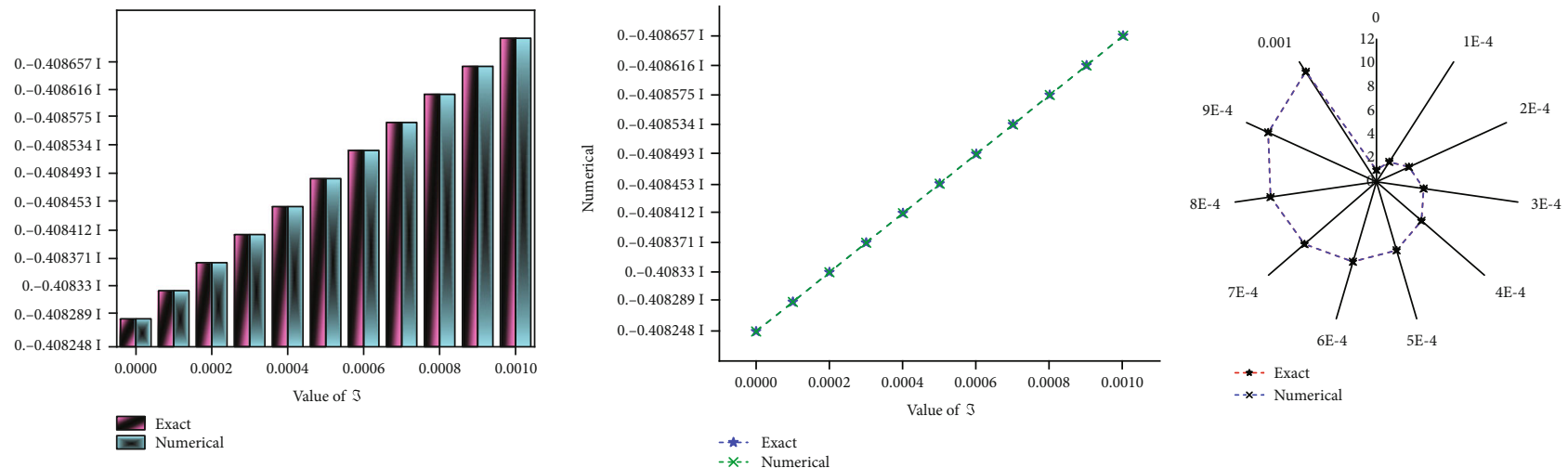

Figure 1: Exact, and numerical solutions based on the obtained analytical solution Equation (16) and septic B-spline scheme.
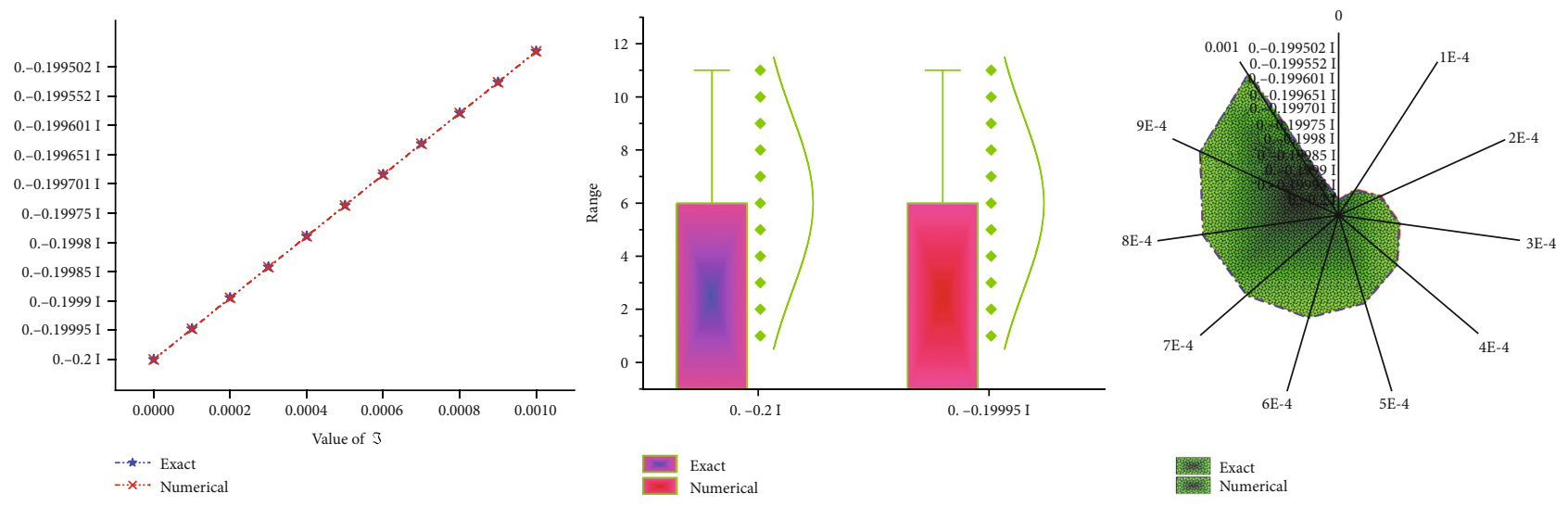

FIGURE 2: Exact and numerical solutions based on the obtained analytical solution Equation (19) and septic B-spline scheme.
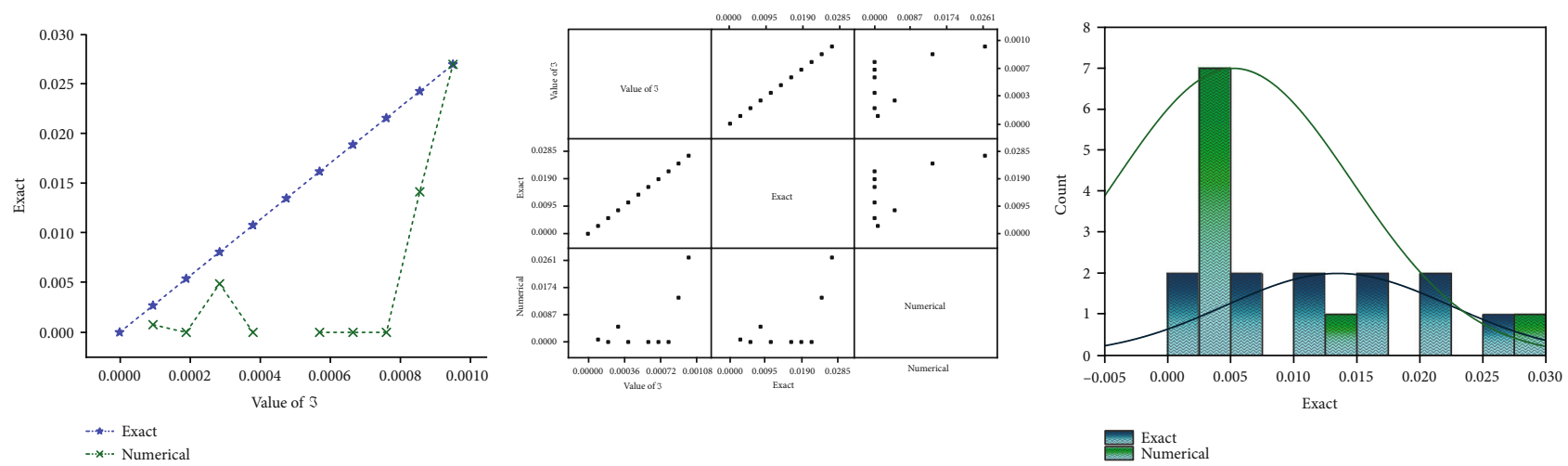

Figure 3: Exact and numerical solutions based on the obtained analytical solution Equation (20) and septic B-spline scheme.

same steps to other obtained solutions investigates their stability property.

\section{Result and Discussion}

Here, we discuss our obtained solutions of the Atangana conformable derivative $(1+3)$-ZK equation with power-law nonlinearity that have been obtained through one of the most recent computational schemes in nonlinear evolution equation field (the mK method) via two main axes which are a compar- ison between our obtained computational solutions and other previous obtained solutions, while the second axis of this discussion is studying our exact and numerical solutions.

(i) Computational solutions

(1) Applying the modified Khater method to the Atangana conformable derivative $(1+3)$ - ZK equation with power-law nonlinearity has obtained sixty distinct traveling wave solutions 
(2) The difference between our obtained solutions and that have been obtained in [41] by Aminikhah et al. who had used the functional variable method; however, they have just found three solutions and accurate in their and our solutions, we can figure out the complete difference between these solutions that thing makes our solutions are novel

(ii) Numerical solutions

(1) Applying the septic B-spline scheme to the Atangana conformable derivative $(1+3)$-ZK equation with power-law nonlinearity by using three of our obtained solutions in evaluating the initial and boundary conditions that give the ability of employing the septic B-spline scheme to the fractional model

\section{Figure and Table Interpretation}

This section illustrates our explained Figures 1-3 and Tables 1-3 with the abovementioned values of the parameters.

(i) Figure 1 and Table 1 show the value of the exact and numerical solutions and absolute error of Equation (5) with Equation (16) in three distinct types of sketches to explain the convergence between the two types of solutions

(ii) Figure 2 and Table 2 show the value of exact and numerical solutions and absolute error of Equation (5) with Equation (19) in three distinct types of sketches to illustrate the closer between the two types of solutions

(iii) Figure 3 and Table 3 explain the value of exact, numerical solutions and absolute error of Equation (5) with Equation (20) in three distinct types of sketches to show the matching between the two types of solutions

\section{Conclusion}

This paper has succeeded in the implementation of the $\mathrm{mK}$ method and septic B-spline scheme to the Atangana conformable derivative $(1+3)$ - ZK equation with power-law nonlinearity. Sixty distinct novel computational solutions have been obtained. Three of these solutions have been used to evaluate the initial and boundary conditions that have allowed the application of the numerical scheme. Calculating the absolute value of error between the exact and numerical is the aim of our study. Moreover, the stability of our obtained solutions has been illustrated based on the Hamiltonian system characteristics. The effectiveness and power of our two used schemes have been verified, and all obtained solutions have been also verified by putting them back in the original equation via Mathematica 12 software.
TABLE 1: Exact and numerical value of the Atangana conformable derivative $(1+3)$-ZK equation with power-law nonlinearity through the obtained analytical solutions via the modified Khater method Equation (16) and septic B-spline scheme.

\begin{tabular}{lccc}
\hline Value of $\mathfrak{F}$ & Exact & Numerical & Absolute error \\
\hline 0 & $0 .-0.408248 \mathrm{I}$ & $0 .-0.0000110311 \mathrm{I}$ & 0.408237 \\
0.0001 & $0 .-0.408289 \mathrm{I}$ & $0 .-8.37587 \times 10^{-6} \mathrm{I}$ & 0.408281 \\
0.0002 & $0 .-0.40833 \mathrm{I}$ & $0 .-5.50221 \times 10^{-6} \mathrm{I}$ & 0.408324 \\
0.0003 & $0 .-0.408371 \mathrm{I}$ & $0 .-2.53611 \times 10^{-6} \mathrm{I}$ & 0.408368 \\
0.0004 & $0 .-0.408412 \mathrm{I}$ & $0 .-1.13958 \times 10^{-6} \mathrm{I}$ & 0.408411 \\
0.0005 & $0 .-0.408453 \mathrm{I}$ & $0 .+1.58999 \times 10^{-7} \mathrm{I}$ & 0.408453 \\
0.0006 & $0 .-0.408493 \mathrm{I}$ & $0 .-1.01297 \times 10^{-6} \mathrm{I}$ & 0.408492 \\
0.0007 & $0 .-0.408534 \mathrm{I}$ & $0 .-2.41857 \times 10^{-6} \mathrm{I}$ & 0.408532 \\
0.0008 & $0 .-0.408575 \mathrm{I}$ & $0 .-5.26488 \times 10^{-6} \mathrm{I}$ & 0.40857 \\
0.0009 & $0 .-0.408616 \mathrm{I}$ & $0 .-7.96518 \times 10^{-6} \mathrm{I}$ & 0.408608 \\
0.001 & $0 .-0.408657 \mathrm{I}$ & $0 .-0.0000103138 \mathrm{I}$ & 0.408647 \\
\hline
\end{tabular}

TABLE 2: Exact and numerical value of the Atangana conformable derivative $(1+3)$-ZK equation with power-law nonlinearity through the obtained analytical solutions via the modified Khater method Equation (19) and septic B-spline scheme.

\begin{tabular}{lccc}
\hline Value of $\mathfrak{F}$ & Exact & Numerical & Absolute error \\
\hline 0 & $0 .-0.2 \mathrm{I}$ & $0 .-0.0000794823 \mathrm{I}$ & 0.199921 \\
0.0001 & $0 .-0.19995 \mathrm{I}$ & $0 .-0.0000595992 \mathrm{I}$ & 0.19989 \\
0.0002 & $0 .-0.1999 \mathrm{I}$ & $0 .-0.0000399605 \mathrm{I}$ & 0.19986 \\
0.0003 & $0 .-0.19985 \mathrm{I}$ & $0 .-0.0000214 \mathrm{I}$ & 0.199829 \\
0.0004 & $0 .-0.1998 \mathrm{I}$ & $0 .-8.76169 \times 10^{-6} \mathrm{I}$ & 0.199792 \\
0.0005 & $0 .-0.19975 \mathrm{I}$ & $0 .+1.47924 \times 10^{-6} \mathrm{I}$ & 0.199752 \\
0.0006 & $0 .-0.199701 \mathrm{I}$ & $0 .-7.5007 \times 10^{-6} \mathrm{I}$ & 0.199693 \\
0.0007 & $0 .-0.199651 \mathrm{I}$ & $0 .-0.0000199929 \mathrm{I}$ & 0.199631 \\
0.0008 & $0 .-0.199601 \mathrm{I}$ & $0 .-0.0000376969 \mathrm{I}$ & 0.199564 \\
0.0009 & $0 .-0.199552 \mathrm{I}$ & $0 .-0.000056177 \mathrm{I}$ & 0.199495 \\
0.001 & $0 .-0.199502 \mathrm{I}$ & $0 .-0.0000736582 \mathrm{I}$ & 0.199428 \\
\hline
\end{tabular}

TABLE 3: Exact and numerical value of the Atangana conformable derivative $(1+3)$-ZK equation with power-law nonlinearity through the obtained analytical solutions via the modified Khater method Equation (20) and septic B-spline scheme.

\begin{tabular}{lccc}
\hline Value of $\mathfrak{F}$ & Exact & Numerical & Absolute error \\
\hline 0 & 0 & $3.46945 \times 10^{-17}$ & $3.46945 \times 10^{-17}$ \\
0.0001 & 0.0027 & 0.000779236 & 0.00192076 \\
0.0002 & 0.0054 & $-8.67362 E-18$ & 0.0054 \\
0.0003 & 0.0081 & 0.00489544 & 0.00320455 \\
0.0004 & 0.0108 & 0 & 0.0108 \\
0.0005 & 0.0135 & $6.50521 E-19$ & 0.0135 \\
0.0006 & 0.0162 & 0 & 0.0162 \\
0.0007 & 0.0189 & $-4.33681 E-19$ & 0.0189 \\
0.0008 & 0.0216 & $-8.67362 E-19$ & 0.0216 \\
0.0009 & 0.0242999 & 0.0141565 & 0.0101435 \\
0.001 & 0.0269999 & 0.0269999 & $3.46945 \times 10^{-18}$ \\
\hline
\end{tabular}




\section{Data Availability}

No data were used to support this study.

\section{Conflicts of Interest}

The authors declare that there is no conflict of interests regarding the publication of this article.

\section{Acknowledgments}

The work was supported by the Natural Science Foundation of China (Grant Nos. 61673169, 11301127, 11701176, 11626101, and 11601485).

\section{References}

[1] A. Lotekar, A. Kakad, and B. Kakad, "Generation of ion acoustic solitary waves through wave breaking in superthermal plasmas," Physics of Plasmas, vol. 24, no. 10, 2017.

[2] K. Devi, A. Nag, J. Paul, and P. K. Karmakar, "Dynamics of sheath evolution in magnetized chargeuctuating dusty plasmas," Chinese Journal of Physics, vol. 65, pp. 405-411, 2020.

[3] P. Ankiewicz, "Perceptions and attitudes of pupils towards technology: In search of a rigorous theoretical framework," International Journal of Technology and Design Education, vol. 29, no. 1, pp. 37-56, 2019.

[4] M.-J. Lee, N. Ashikawa, and Y.-D. Jung, "Characteristics of ion-cyclotron surface waves in semi-bounded (r, q) distribution dusty plasmas," Physics of Plasmas, vol. 25, article 062110, no. 6, 2018.

[5] M. M. Hossen, M. S. Alam, S. Sultana, and A. Mamun, "Low frequency nonlinear waves in electron depleted magnetized nonthermal plasmas," The European Physical Journal D, vol. 70, no. 12, 2016.

[6] F. S. Khodadad, F. Nazari, M. Eslami, and H. Rezazadeh, "Soliton solutions of the conformable fractional ZakharovKuznetsov equation with dual-power law nonlinearity," Optical and Quantum Electronics, vol. 49, no. 11, 2017.

[7] M. M. Khater, R. A. Attia, A.-H. Abdel-Aty, W. Alharbi, and D. Lu, "Abundant analytical and numerical solutions of the fractional microbiological densities model in bacteria cell as a result of diffusion mechanisms," Solitons \& Fractals, vol. 136, 2020.

[8] Z. Korpinar, M. Inc, and M. Bayram, "Theory and application for the system of fractional Burger equations with Mittag leffler kernel," Applied Mathematics and Computation, vol. 367, 2020.

[9] C. Park, M. M. Khater, A.-H. Abdel-Aty et al., "Dynamical analysis of the nonlinear complex fractional emerging telecommunication model with higher-order dispersive cubicquintic," Alexandria Engineering Journal, vol. 59, no. 3, pp. 1425-1433, 2020.

[10] M. A. Abdelrahman, S. I. Ammar, K. M. Abualnaja, and M. Inc, "New solutions for the unstable nonlinear Schrödinger equation arising in natural science," Aims Mathematics, vol. 5, no. 3, pp. 1893-1912, 2020.

[11] M. M. Khater, B. Ghanbari, K. S. Nisar, and D. Kumar, "Novel exact solutions of the fractional Bogoyavlensky-Konopelchenko equation involving the Atangana-Baleanu-Riemann derivative," Alexandria Engineering Journal, 2020, In press.
[12] Z.-y. Zhang, Z.-h. Liu, X.-j. Miao, and Y.-z. Chen, "New exact solutions to the perturbed nonlinear Schrödinger's equation with kerr law nonlinearity," Applied Mathematics and Computation, vol. 216, no. 10, pp. 3064-3072, 2010.

[13] Z.-y. Zhang, Y.-x. Li, Z.-h. Liu, and X.-j. Miao, "New exact solutions to the perturbed nonlinear Schrodinger's equation with kerr law nonlinearity via modi_ed trigonometric function series method," Communications in Nonlinear Science and Numerical Simulation, vol. 16, no. 8, pp. 3097-3106, 2011.

[14] Z.-y. Zhang, Z.-h. Liu, X.-j. Miao, and Y.-z. Chen, "Qualitative analysis and traveling wave solutions for the perturbed nonlinear Schrödinger's equation with kerr law nonlinearity," Physics Letters A, vol. 375, no. 10, pp. 1275-1280, 2011.

[15] X.-j. Miao and Z.-y. Zhang, “The modified $G^{\prime} / G$-expansion method and traveling wave solutions of nonlinear the perturbed nonlinear Schrödinger's equation with kerr law nonlinearity," Communications in Nonlinear Science and Numerical Simulation, vol. 16, no. 11, pp. 4259-4267, 2011.

[16] A.-H. Abdel-Aty, M. Khater, R. A. Attia, and H. Eleuch, "Exact traveling and nano-solitons wave solitons of the ionic waves propagating along microtubules in living cells," Mathematics, vol. 8, no. 5, 2020.

[17] J. Li, R. A. Attia, M. M. Khater, and D. Lu, "The new structure of analytical and semi-analytical solutions of the longitudinal plasma wave equation in a magneto-electro-elastic circular rod," Modern Physics Letters B, vol. 34, article 2050123, no. $12,2020$.

[18] M. M. Khater, R. A. Attia, S. S. Alodhaibi, and D. Lu, "Novel soliton waves of two uid nonlinear evolutions models in the view of computational scheme," International Journal of Modern Physics B, vol. 34, article 2050096, no. 10, 2020.

[19] H. Rezazadeh, D. Kumar, A. Neirameh, M. Eslami, and M. Mirzazadeh, "Applications of three methods for obtaining optical soliton solutions for the Lakshmanan-Porsezian-Daniel model with Kerr law nonlinearity," Pramana, vol. 94, no. 1, 2020.

[20] S. M. Mirhosseini-Alizamini, H. Rezazadeh, M. Eslami, M. Mirzazadeh, and A. Korkmaz, "New extended direct algebraic method for the Tzitzica type evolution equations arising in nonlinear optics," Computational Methods for Differential Equations, vol. 8, no. 1, pp. 28-53, 2020.

[21] Z.-Y. Zhang, X.-Y. Gan, and D.-M. Yu, "Bifurcation Behaviour of the Travelling Wave Solutions of the Perturbed Nonlinear Schrödinger Equation with Kerr Law Nonlinearity," Zeitschrift für Naturforschung A, vol. 66, no. 12, pp. 721-727, 2011.

[22] Z. Zhang, J. Huang, J. Zhong et al., "The extended $\left(G^{\prime} / G\right)$ expansion method and travelling wave solutions for the perturbed nonlinear Schrödinger's equation with Kerr law nonlinearity," Pramana, vol. 82, no. 6, pp. 1011-1029, 2014.

[23] Z. Zai-Yun, G. Xiang-Yang, Y. De-Min, Z. Ying-Hui, and L. Xin-Ping, "A Note on Exact Traveling Wave Solutions of the Perturbed Nonlinear Schrödinger's Equation with Kerr Law Nonlinearity," Communications in Theoretical Physics, vol. 57, no. 5, p. 764, 2012.

[24] A. Korkmaz and K. Hosseini, "Exact solutions of a nonlinear conformable time-fractional parabolic equation with exponential nonlinearity using reliable methods," Optical and Quantum Electronics, vol. 49, no. 8, 2017.

[25] S. M. Mirhosseini-Alizamini, H. Rezazadeh, K. Srinivasa, and A. Bekir, "New closed form solutions of the new coupled Konno-Oono equation using the new extended direct algebraic method," Pramana, vol. 94, no. 1, 2020. 
[26] C. Yue, A. Elmoasry, M. Khater et al., "On complex wave structures related to the nonlinear long-short wave interaction system: analytical and numerical techniques," AIP Advances, vol. 10, no. 4, 2020.

[27] M. M. Khater, R. A. Attia, and D. Lu, "Computational and numerical simulations for the nonlinear fractional Kolmogorov-Petrovskii-Piskunov (FKPP) equation," Physica Scripta, vol. 95, no. 5, 2020.

[28] N. A. Kudryashov, "Periodic and solitary waves of the BiswasArshed equation," Optik, vol. 200, 2020.

[29] M. Torvattanabun, P. Juntakud, A. Saiyun, and N. Khansai, "The new exact solutions of the new coupled Konno-Oono equation by using extended simplest equation method," Applied Mathematical Sciences, vol. 12, no. 6, pp. 293-301, 2018.

[30] M. Kaplan, A. Bekir, and A. Akbulut, “A generalized Kudryashov method to some nonlinear evolution equations in mathematical physics," Nonlinear Dynamics, vol. 85, no. 4, pp. 2843-2850, 2016.

[31] M. M. Khater, D. Lu, and E. H. Zahran, "Solitary Wave Solutions of the Benjamin-Bona- Mahoney-Burgers Equation with Dual Power-Law Nonlinearity," Applied Mathematics \& Information Sciences, vol. 11, no. 5, pp. 1-5, 2017.

[32] K. Hosseini, E. Y. Bejarbaneh, A. Bekir, and M. Kaplan, "New exact solutions of some nonlinear evolution equations of pseudoparabolic type," Optical and Quantum Electronics, vol. 49, no. 7, 2017.

[33] H. Gündoğdu and Ö. F. Gözükızıl, "Applications of the decomposition methods to some nonlinear partial differential equations," New Trends in Mathematical Sciences, vol. 6, no. 3, pp. 57-66, 2018.

[34] O. A. Ilhan, A. Esen, H. Bulut, and H. M. Baskonus, "Singular solitons in the pseudo-parabolic model arising in nonlinear surface waves," Results in Physics, vol. 12, pp. 1712-1715, 2019.

[35] L. Á valos-Ruiz, J. Go' mez-Aguilar, A. Atangana, and K. M. Owolabi, "On the dynamics of fractional maps with powerlaw, exponential decay and Mittag-Leffler memory," Chaos, Solitons \& Fractals, vol. 127, pp. 364-388, 2019.

[36] M. N. Alam and C. Tunc, "Constructions of the optical solitons and other solitons to the conformable fractional Zakharov-Kuznetsov equation with power law nonlinearity," Journal of Taibah University for Science, vol. 14, no. 1, pp. 94-100, 2020.

[37] M. Osman, H. Rezazadeh, and M. Eslami, "Traveling wave solutions for $(3+1)$ dimensional conformable fractional Zakharov-Kuznetsov equation with power law nonlinearity," Nonlinear Engineering, vol. 8, no. 1, pp. 559-567, 2019.

[38] Q. Jin, T. Xia, and J. Wang, "The Exact Solution of the SpaceTime Fractional Modified Kdv-Zakharov-Kuznetsov Equation," Journal of Applied Mathematics and Physics, vol. 5, no. 4, pp. 844-852, 2017.

[39] H. Yépez-Martínez and J. F. Gómez-Aguilar, "Fractional subequation method for Hirota-Satsuma-coupled KdV equation and coupled $\mathrm{mKdV}$ equation using the Atangana's conformable derivative," Waves in Random and Complex Media, vol. 29, no. 4, pp. 678-693, 2019.
[40] H. Yepez-Martinez and J. Gomez-Aguilar, "Optical solitons solution of resonance nonlinear Schrödinger type equation with Atangana's-conformable derivative using sub-equation method," Waves in Random and Complex Media, pp. 1-24, 2019, In press.

[41] H. Aminikhah, B. P. Ziabary, and H. Rezazadeh, "Exact traveling wave solutions of partial differential equations with power law nonlinearity," Nonlinear Engineering, vol. 4, no. 3, 2015. 